\title{
Study of Kenmotsu manifolds with semi-symmetric metric connection
}

\author{
Sudhakar Kr Chaubey ${ }^{\mathrm{a}^{*}}$ and Sunil Kr Yadav \\ ${ }^{a}$ Section of Mathematics, Department of Information Technology, Shinas College of Technology, Shinas, P.O. Box 77 Postal Code 324 , Oman \\ ${ }^{\mathrm{b}}$ Department of Mathematics, Poornima college of Engineering, Jaipur-302022, Rajasthan, India \\ *Corresponding authorE-mail: sk22_math@yahoo.co.in; sudhakar.chaubey@shct.edu.om
}

\author{
Article Info \\ Keywords: Codazzi type, Cyclic par- \\ allel Ricci tensors, Different curva- \\ ture tensors, $\eta$-parallel Ricci tensor, \\ Kenmotsu manifold, Symmetric spaces, \\ Semi-symmetric metric connection \\ 2010 AMS: 53B15, 53C55, 53C12, \\ 53C40, 53C42. \\ Received: 25 May 2018 \\ Accepted: 20 June 2018 \\ Available online: 26 June 2018
}

\begin{abstract}
The present paper deals with the study of Kenmotsu manifolds equipped with a semisymmetric metric connection. The properties of $\eta$-parallel Ricci tensor, globally symmetric and $\phi$-symmetric Kenmotsu manifolds with the semi-symmetric metric connection are evaluated. In the end, we construct an example of a 3-dimensional Kenmotsu manifold admitting semi-symmetric metric connection and verify our some results.
\end{abstract}

\section{Introduction}

The notion of curvatures play a central role in the differential geometry and physics. For instant, the magnitude of a force required to move an object at constant speed along a curve path is, according to Newton's laws, a constant multiple of the curvature of the trajectory. The motion of a body in a gravitational field is determined, according to Einstein, by the curvature of spacetime [1]. The space of constant curvature also plays a key role in differential geometry and mathematical physics (specially in the theory of relativity and cosmology). In 1926, Cartan ([2], [3]) introduced the notion of a locally symmetric Riemannian manifold which is the natural generalization of manifolds of constant curvature. The condition of local symmetry is equivalent to the fact that at every point $x \in M$, the local geodesic symmetry $F(x)$ is an isometry [4]. The idea of locally $\phi$-symmetric Sasakian manifold was introduced by Takahashi [35] in 1977. Since then, the properties of such manifolds have been studied by several geometers on different spaces.

The study of odd dimensional manifolds with contact and almost contact structures were initiated by Boothby and Wong [5] in 1958 rather from topological point of view. Sasaki and Hatakeyama [6] re-investigated them using tensor calculus in 1961. In 1972, K. Kenmotsu studied a class of almost contact metric manifolds and call them Kenmotsu manifold [7]. He proved that if a Kenmotsu manifold satisfies the condition $R(X, Y) \cdot R=0$, then the manifold is of negative curvature -1 , where $R$ is the Riemannian curvature tensor of type $(1,3)$ and $R(X, Y)$ denotes the derivation of the tensor algebra at each point of the tangent space $T(M)$. The properties of Kenmotsu manifolds have been noticed in ([9] - [17]) and by others.

The notion of a semi-symmetric linear connection on a differentiable manifold has been introduced by Friedmann and Schouten [20] in 1924. Hayden [21] in 1932, introduced and studied the idea of semi-symmetric linear connection with torsion on a Riemannian manifold. After a long interval, Yano [22] started the systematic study of a semi-symmetric metric connection on a Riemannian manifold in 1970. Since then the properties of semi-symmetric metric connection on different spaces have studied in ([27]-[32]) and the references therein.

Motivated from the above studied, authors start the study of the properties of Kenmotsu manifold equipped with a semi-symmetric metric connection. We organize the present paper as follows: Section 2 contains the basic known results of Kenmotsu manifolds and $\eta-$ parallel Ricci tensor. The brief results of the semi-symmetric metric connection on a Kenmotsu manifold are given in section 3. Section 4 deals the study of $\eta$-parallel Ricci tensor with respect to the semi-symmetric metric connection on the Kenmotsu manifold and find some geometrical results. The properties of concircular and projective curvature tensors endowed with a semi-symmetric metric connection are investigated in section 5. In last section, we construct an example of Kenmotsu manifold equipped with semi-symmetric connection and verify our results. 


\section{Preliminaries}

An odd dimensional differentiable manifold $M(\operatorname{dim} M=n=2 m+1)$ of class $C^{\infty}$ is said to have a $(\phi, \xi, \eta)$-structure or almost contact structure if it admits a tensor field $\phi$ of endomorphisms of the tangent spaces, a vector field $\xi$, and a $1-$ form $\eta$ satisfying

$$
\eta(\xi)=1 \text { and } \phi^{2}=-I+\eta \otimes \xi,
$$

where $I$ denotes the identity transformation [25]. From (2.1), it can be easily see that $\phi \xi=0, \eta \circ \phi=0$ and rank $\phi=n-1$. A Riemannian metric $g$ of type $(0,2)$ is said to be compatible with the almost contact structure $(\phi, \xi, \eta)$ if the relation

$$
g(X, Y)=g(\phi X, \phi Y)+\eta(X) \eta(Y)
$$

holds for arbitrary vector fields $X$ and $Y$. An almost contact structure $(\phi, \xi, \eta)$ equipped with a compatible Riemannian metric $g$ is known as almost contact metric structure $(\phi, \xi, \eta, g)$ and the manifold $M$ endowed with the almost contact structure is called an almost contact metric manifold. If moreover,

$$
\nabla_{X} \xi=X-\eta(X) \xi
$$

holds for all $X$ on $M(\phi, \xi, \eta, g)$, then the manifold is said to be Kenmotsu manifold [7]. Here $\nabla$ denotes the Levi-Civita connection of the metric $g$. For proving our main results in next sections, we are going to recall some basic known results of Kenmotsu manifold as:

$$
\left(\nabla_{X} \phi\right)(Y)=-\eta(Y) \phi X-g(X, \phi Y) \xi
$$

$$
\left(\nabla_{X} \eta\right)(Y)=g(X, Y)-\eta(X) \eta(Y)
$$

$$
S(\phi X, \phi Y)=S(X, Y)+(n-1) \eta(X) \eta(Y)
$$

$$
\eta(R(X, Y) Z)=\eta(Y) g(X, Z)-\eta(X) g(Y, Z)
$$

$$
R(X, Y) \xi=\eta(X) Y-\eta(Y) X
$$

$$
R(\xi, X) Y=\eta(Y) X-g(X, Y) \xi
$$

$$
S(X, \xi)=-(n-1) \eta(X) .
$$

A Kenmotsu manifold $M$ is said to be $\eta$-Einstein if its Ricci tensor $S$ takes the form

$$
S(X, Y)=a g(X, Y)+b \eta(X) \eta(Y),
$$

for arbitrary vector fields $X$ and $Y$, where $a$ and $b$ are smooth functions on $(M, g)$ [7]. If $b=0$, then $\eta$-Einstein manifold becomes Einstein manifold. It is well known that in a Kenmotsu manifold $a+b=-(n-1)$ (see [7], p. 97).

The notion of $\eta$-parallelism on a Sasakian manifold was introduced by M. Kon [26]. A Ricci tensor $S$ of an $n$-dimensional Kenmotsu manifold $M$ is said to be $\eta$-parallel if it satisfies the tensorial relation

$$
\left(\nabla_{X} S\right)(\phi Y, \phi Z)=0
$$

for all $X, Y, Z \in \chi(M)$.

\section{Semi-symmetric metric connection}

Let $M$ be an $n$-dimensional Kenmotsu manifold and $\nabla$ denotes the Levi-Civita connection on it. A linear connection $\tilde{\nabla}$ on $M$ is said to be a semi-symmetric if the torsion tensor $\tilde{T}$ of type $(1,2)$ defined as

$$
\tilde{T}(X, Y)=\tilde{\nabla}_{X} Y-\tilde{\nabla}_{Y} X-[X, Y]
$$

satisfies

$$
\tilde{T}(X, Y)=\eta(Y) X-\eta(X) Y
$$

for all vector fields $X$ and $Y$ on $M$. If moreover, the semi-symmetric connection $\tilde{\nabla}$ holds the relation

$$
\tilde{\nabla} g=0
$$


is called semi-symmetric metric connection. A semi-symmetric connection $\tilde{\nabla}$ is said to be non-metric if $\tilde{\nabla} g \neq 0$. A relation between a semi-symmetric metric and Levi-Civita connections is given by the relation

$$
\tilde{\nabla}_{X} Y=\nabla_{X} Y+\eta(Y) X-g(X, Y) \xi
$$

for all vector fields $X, Y \in \chi(M)$ ([22], p. 15). With the help of equations (2.1), (2.2), (2.3), (2.4) and (3.3), we can easily observe that

$$
\left(\tilde{\nabla}_{X} \eta\right)(Y)=\left(\nabla_{X} \eta\right)(Y)-\eta(X) \eta(Y)+g(X, Y)
$$

and

$$
\left(\tilde{\nabla}_{X} \phi\right)(Y)=-g(X, \phi Y) \xi-2 \eta(Y) \phi X \text {. }
$$

If $R$ and $\tilde{R}$ denote the curvature tensors with respect to the Levi-Civita and semi-symmetric metric connections of the manifold $M$ respectively, then it is related by the relation

$$
\tilde{R}(X, Y) Z=R(X, Y) Z+\theta(X, Z) Y-\theta(Y, Z) X+g(X, Z) L Y-g(Y, Z) L X
$$

for all $X, Y, Z \in \chi(M)$, where

$$
\theta(X, Y)=g(L X, Y)=\left(\nabla_{X} \eta\right)(Y)-\eta(X) \eta(Y)+\frac{1}{2} g(X, Y)
$$

is a symmetric tensor of type $(0,2)$ on $M$. In consequence of (2.1), (2.5) and (3.7), equation (3.6) assumes the form

$$
\tilde{R}(X, Y) Z=R(X, Y) Z-3 g(Y, Z) X+3 g(X, Z) Y+2 \eta(Y) \eta(Z) X-2 \eta(X) \eta(Z) Y+2 \eta(X) g(Y, Z) \xi-2 \eta(Y) g(X, Z) \xi .
$$

The contraction of equation (3.8) along the vector field $X$ gives

$$
\tilde{S}(Y, Z)=S(Y, Z)-(3 n-5) g(Y, Z)+2(n-2) \eta(Y) \eta(Z),
$$

which is equivalent to

$$
\tilde{Q} Y=Q Y-(3 n-5) Y+2(n-2) \eta(Y) \xi \text {, }
$$

where $\tilde{Q}$ and $Q$ denote the Ricci operators corresponding to the connections $\tilde{\nabla}$ and $\nabla$ respectively and defined as $\tilde{S}(Y, Z)=g(\tilde{Q} Y, Z)$ and $S(Y, Z)=g(Q Y, Z)$. Let $\left\{e_{i}, i=1,2,3, \ldots, n\right\}$ be an orthonormal basis of the tangent space at each point of the manifold $M$. Setting $Y=Z=e_{i}$ in (3.9) and taking summation over $i, 1 \leq i \leq n$, we get

$$
\tilde{r}=r-n(3 n-7)-4,
$$

where

$$
\tilde{r}=\sum_{i=1}^{n} \tilde{S}\left(e_{i}, e_{i}\right) \quad \text { and } \quad r=\sum_{i=1}^{n} S\left(e_{i}, e_{i}\right)
$$

represent the scalar curvatures with respect to the connections $\tilde{\nabla}$ and $\nabla$ respectively. In view of equations (2.1), (2.10) and (3.9), we can find that

$$
\tilde{S}(Y, \xi)=-2(n-1) \eta(Y) .
$$

With the help of (2.1), (2.8), (2.9) and (3.8), we can easily calculate the following:

$$
\tilde{R}(\xi, Y) Z=2\{\eta(Z) Y-g(Y, Z) \xi\}
$$

and

$$
\tilde{R}(X, Y) \xi=2\{\eta(X) Y-\eta(Y) X\} .
$$

The equation (3.14) shows that the manifold $M$ equipped with $\tilde{\nabla}$ is regular.

\section{4. $\eta$-parallel Ricci tensor with respect to semi-symmetric metric connection}

In this section, we study the geometrical properties of $\eta$-parallel Ricci tensor with respect to the semi-symmetric metric connection $\tilde{\nabla}$. In [8], authors studied the properties of $\eta$-parallel Ricci tensor and proved several results. Analogous to the definition of $\eta$-parallelism given by M. Kon [26] on Sasakian manifolds, we define

Definition 4.1. A Ricci tensor $\tilde{S}$ of an $n$-dimensional Kenmotsu manifold $M$ endowed with a semi-symmetric metric connection $\tilde{\nabla}$ is said to be $\eta$-parallel for $\tilde{\nabla}$ if it satisfies the relation $\left(\tilde{\nabla}_{X} \tilde{S}\right)(\phi Y, \phi Z)=0$, for arbitrary vector fields $X, Y$ and $Z$. 
From (3.3), it is obvious that

$$
\tilde{\nabla}_{X}(\tilde{Q} Y)=\nabla_{X}(\tilde{Q} Y)+g(\tilde{Q} Y, \xi) X-g(X, \tilde{Q} Y) \xi .
$$

With the help of (2.1), (2.5), (3.3) and (3.10), we can find

$$
\tilde{\nabla}_{X}(\tilde{Q} Y)=\left(\tilde{\nabla}_{X} \tilde{Q}\right)(Y)+Q\left(\nabla_{X} Y\right)+2(n-1) g(X, Y) \xi+\eta(Y) Q X+2(n-2)\left\{\eta\left(\nabla_{X} Y\right)-\eta(X) \eta(Y)\right\} \xi-(3 n-5)\left\{\nabla_{X} Y+\eta(Y) X\right\},
$$

and

$$
\nabla_{X}(\tilde{Q} Y)=\left(\nabla_{X} Q\right)(Y)+Q\left(\nabla_{X} Y\right)-(3 n-5) \nabla_{X} Y+2(n-2)\left[\left\{\eta\left(\nabla_{X} Y\right)-\eta(X) \eta(Y)+g(X, Y)\right\} \xi+\eta(Y)\{X-\eta(X) \xi\}\right] .
$$

From the above results, we obtain

$$
\left(\tilde{\nabla}_{X} \tilde{Q}\right)(Y)=\left(\nabla_{X} Q\right)(Y)-\eta(Y) Q X-g(X, Q Y) \xi-8(n-2) \eta(Y) \eta(X) \xi+(3 n-7)\{\eta(Y) X+g(X, Y) \xi\} .
$$

In view of $(2.1),(2.10)$ and $\left(\tilde{\nabla}_{X} \tilde{S}\right)(Y, Z)=g\left(\left(\tilde{\nabla}_{X} \tilde{Q}\right)(Y), Z\right)$, above relation assumes the form

$$
\left(\tilde{\nabla}_{X} \tilde{S}\right)(Y, Z)=\left(\nabla_{X} S\right)(Y, Z)-\eta(Y) S(X, Z)-\eta(Z) S(X, Y)+(3 n-7)\{\eta(Z) g(X, Y)+\eta(Y) g(X, Z)\}-8(n-2) \eta(X) \eta(Y) \eta(Z) .
$$

Replacing the vector fields $Y$ by $\phi Y$ and $Z$ by $\phi Z$ in (??) and then using (2.1), we obtain

$$
\left(\tilde{\nabla}_{X} \tilde{S}\right)(\phi Y, \phi Z)=\left(\nabla_{X} S\right)(\phi Y, \phi Z) \text {. }
$$

In view of (2.12), (4.1) and Definition 4.1, we can state the following:

Theorem 4.2. Let $M$ be an $n$-dimensional Kenmotsu manifold equipped with a semi-symmetric metric connection $\tilde{\nabla}$. Then the Ricci tensor $\tilde{S}$ on $M$ is $\eta$-parallel with respect to the connection $\tilde{\nabla}$ if and only if the manifold has $\eta$-parallel Ricci tensor S for the Levi-Civita connection $\nabla$.

The straight forward calculations from the equations (2.1), (2.2), (2.6) and (3.9) give

$$
\tilde{S}(\phi Y, \phi Z)=\tilde{S}(Y, Z)+2(n-1) \eta(Y) \eta(Z)
$$

for all $X, Y \in \chi(M)$. Differentiating (4.2) covariantly along the vector field $X$, we have

$$
\left(\tilde{\nabla}_{X} \tilde{S}\right)(\phi Y, \phi Z)=\tilde{\nabla}_{X} \tilde{S}(\phi Y, \phi Z)-\tilde{S}\left(\tilde{\nabla}_{X}(\phi Y), \phi Z\right)-\tilde{S}\left(\phi Y, \tilde{\nabla}_{X}(\phi Z)\right) .
$$

With the help of equations (2.1), (3.3), (3.4), (3.5), (3.12) and (4.2), last equation assumes the form

$$
\left(\tilde{\nabla}_{X} \tilde{S}\right)(\phi Y, \phi Z)=\left(\tilde{\nabla}_{X} \tilde{S}\right)(Y, Z)+2\{\eta(Y) \tilde{S}(X, Z)+\eta(Z) \tilde{S}(X, Y)\}+4(n-1)\{\eta(Y) g(X, Z)+\eta(Z) g(X, Y)\} .
$$

Let us suppose that the manifold $M$ equipped with a semi-symmetric metric connection $\tilde{\nabla}$ has $\eta$-parallel Ricci tensor $\tilde{S}$ for the connection $\tilde{\nabla}$, i.e. $\left(\tilde{\nabla}_{X} \tilde{S}\right)(\phi Y, \phi Z)=0$, then from (4.3), we obtain

$$
\left(\tilde{\nabla}_{X} \tilde{S}\right)(Y, Z)=-2\{\eta(Y) \tilde{S}(X, Z)+\eta(Z) \tilde{S}(X, Y)\}-4(n-1)\{\eta(Y) g(X, Z)+\eta(Z) g(X, Y)\} .
$$

Thus, we can state the following:

Theorem 4.3. An $n$-dimensional Kenmotsu manifold $M$ endowed with a semi-symmetric metric connection $\tilde{\nabla}$ has $\eta-$ parallel Ricci tensor for the connection $\tilde{\nabla}$ if and only if the relation (4.4) holds on $M$.

Let $\left\{e_{i}, i=1,2,3, \ldots, n\right\}$ be an orthonormal basis of the tangent space at any point of the manifold $M$. Setting $Y=Z=e_{i}$ in (4.4) and taking summation over $i, 1 \leq i \leq n$, we get

$$
d \tilde{r}(X)=0
$$

for all $X \in \chi(M)$. This shows that the scalar curvature $\tilde{r}$ is constant with respect to the connection $\tilde{\nabla}$. Hence we state:

Corollary 4.4. If the Ricci tensor $\tilde{S}$ of an $n$-dimensional Kenmotsu manifold $M$ equipped with a semi-symmetric metric connection $\tilde{\nabla}$ is $\eta$-parallel, then the scalar curvature for the connection $\tilde{\nabla}$ is constant.

From (3.11) and Corollary 4.4, it is obvious that

$$
d \tilde{r}(X)=d r(X)=0,
$$

$\forall X \in \chi(M)$, which implies that the scalar curvature with respect to the Levi-Civita connection is constant. Thus we have,

Corollary 4.5. Let an $n$-dimensional Kenmotsu manifold M equipped with a semi-symmetric metric connection $\tilde{\nabla}$ has $\eta-$ parallel Ricci tensor $\tilde{S}$. Then the scalar curvature of the manifold is constant.

Moreover, since $\tilde{S}(Y, Z)=g(\tilde{Q} Y, Z)$, we can find from (3.3) that

$$
\tilde{\nabla}_{X}|\tilde{Q}|^{2}=2 \sum_{i=1}^{n} g\left(\left(\tilde{\nabla}_{X} \tilde{Q}\right) e_{i}, \tilde{Q} e_{i}\right)=0
$$

which implies that $|\tilde{Q}|^{2}=$ constant. Thus we have 
Corollary 4.6. If an $n$-dimensional Kenmotsu manifold endowed with a semi symmetric metric connection $\tilde{\nabla}$ has $\eta$-parallel Ricci tensor, then the length of Ricci operator with $\tilde{\nabla}$ is constant on $M$.

Let us suppose that the Ricci tensor $\tilde{S}$ on an $n$-dimensional Kenmotsu manifold $M$ equipped with a semi-symmetric metric connection $\tilde{\nabla}$ is Codazzi type, i.e., $\left(\tilde{\nabla}_{X} \tilde{S}\right)(Y, Z)=\left(\tilde{\nabla}_{Y} \tilde{S}\right)(X, Z)$. Thus in view of this definition and equation (4.4), we obtain

$$
\eta(Y) \tilde{S}(X, Z)-\eta(X) \tilde{S}(Y, Z)=2(n-1)\{\eta(X) g(Y, Z)-\eta(Y) g(X, Z)\} .
$$

Setting $Y=\xi$ in last expression and using (2.1) and (3.12), we find

$$
\tilde{S}(X, Z)=-2(n-1) g(X, Z),
$$

which is equivalent to

$$
S(X, Z)=(n-3) g(X, Z)-2(n-2) \eta(X) \eta(Z) .
$$

This shows that the manifold $M$ is an $\eta$-Einstein manifold with the scalars $a=n-3$ and $b=-2(n-2)$. It is obvious that $a+b=-(n-1)$ [For instant, see [7], p-97]. Conversely, if we suppose that the manifold $M$ satisfies (4.5), then we can easily find that the Ricci tensor with respect to the semi-symmetric metric connection $\tilde{\nabla}$ is of Codazzi type. Thus we can state:

Corollary 4.7. Let an $n(>3)$-dimensional Kenmotsu manifold M equipped with a semi-symmetric metric connection $\tilde{\nabla}$ has $\eta-$ parallel Ricci tensor for $\tilde{\nabla}$. Then the Ricci tensor $\tilde{S}$ on $M$ to be of Codazzi type if and only if the manifold $M$ is Einstein for $\tilde{\nabla}$ or an $\eta-$ Einstein for Levi-Civita connection.

Again, we consider that the Ricci tensor $\tilde{S}$ with respect to a semi-symmetric metric connection $\tilde{\nabla}$ is cyclic parallel, i.e.,

$$
\left(\tilde{\nabla}_{X} \tilde{S}\right)(Y, Z)+\left(\tilde{\nabla}_{Y} \tilde{S}\right)(Z, X)+\left(\tilde{\nabla}_{Z} \tilde{S}\right)(X, Y)=0 .
$$

In view of (4.4), above relation converts into the form

$$
\eta(X) \tilde{S}(Y, Z)+\eta(Y) \tilde{S}(Z, X)+\eta(Z) \tilde{S}(X, Y)+2(n-1)\{\eta(X) g(Y, Z)+\eta(Y) g(X, Z)+\eta(Z) g(X, Y)\}=0 .
$$

Putting $Z=\xi$ in last expression and using (2.1) and (3.12), we get (4.5). Hence we can state:

Corollary 4.8. Let $M$ be an $n(>3)$-dimensional Kenmotsu manifold endowed with a semi-symmetric metric connection $\tilde{\nabla}$, has $\eta$-parallel Ricci tensor $\tilde{S}$, then the Ricci tensor with respect to the connection $\tilde{\nabla}$ to be cyclic parallel if and only if the manifold is $\eta-E i n s t e i n$.

\section{Concircular curvature tensor with semi-symmetric metric connection $\tilde{\nabla}$}

It is well known that a geodesic circle (a curve whose first curvature is constant and second curvature is identically zero) does not transform into a geodesic circle by the conformal transformation

$$
\overline{g_{i j}}=\psi^{2} g_{i j}
$$

where $g_{i j}$ denotes the fundamental tensor. Yano [18] proved that a conformal transformation, defined in (5.1), satisfying the partial differential equation

$$
\psi_{i ; j}=\phi g_{i j}
$$

alters a geodesic circle into a geodesic circle. Such a transformation is known as concircular transformation and the geometry deals with such transformation is called the concircular geometry [18]. A tensor field $C$ of type $(1,3)$ on a Riemannian manifold, which remains invariant under the concircular transformation, defined by

$$
C(X, Y) Z=R(X, Y) Z-\frac{r}{n(n-1)}\{g(Y, Z) X-g(X, Z) Y\},
$$

where $R$ is the curvature tensor and $r$ denotes the scalar curvature, is known as concircular curvature tensor [19]. Analogous to the definition of (5.3), we can define

Definition 5.1. Let $M$ be an $n$-dimensional Kenmotsu manifold equipped with a semi-symmetric metric connection $\tilde{\nabla}$. A concircular curvature tensor $\tilde{C}$ with respect to the connection $\tilde{\nabla}$ on $M$ is a tensor field of type $(1,3)$ and satisfies the relation

$$
\tilde{C}(X, Y) Z=\tilde{R}(X, Y) Z-\frac{\tilde{r}}{n(n-1)}\{g(Y, Z) X-g(X, Z) Y\},
$$

for all vector fields $X, Y, Z \in \chi(M)$. Here $\tilde{R}$ and $\tilde{r}$ are the curvature tensor and scalar curvature of the manifold $M$ corresponding to the connection $\tilde{\nabla}$ respectively.

Definition 5.2. Let $M$ be an $n$-dimensional Kenmotsu manifold equipped with a semi-symmetric metric connection $\tilde{\nabla}$. A projective curvature tensor $\tilde{P}$ with respect to the connection $\tilde{\nabla}$ on $M$ is a tensor field of type $(1,3)$ and satisfies the relation

$$
\tilde{P}(X, Y) Z=\tilde{R}(X, Y) Z-\frac{1}{(n-1)}\{\tilde{S}(Y, Z) X-\tilde{S}(X, Z) Y\},
$$

for all vector fields $X, Y, Z \in \chi(M)$. Here $\tilde{R}$ and $\tilde{S}$ denote the curvature and Ricci tensors of the manifold $M$ corresponding to the connection $\tilde{\nabla}$ respectively. 
Let $M$ be an $n(>3)$-dimensional Kenmotsu manifold admitting a semi-symmetric metric connection $\tilde{\nabla}$ has $\eta$-parallel Ricci tensor $\tilde{S}$ for $\tilde{\nabla}$. If additionally, Ricci tensor $\tilde{S}$ is either Codazzi type or cyclic parallel, then equation (4.5) holds on $M$. Let $\left\{e_{i}, i=1,2,3, \ldots, n\right\}$ be an orthonormal basis of the tangent space at any point of the manifold $M$. Setting $Y=Z=e_{i}$ in (4.5) and taking summation over $i, 1 \leq i \leq n$, we get $\tilde{r}=-2 n(n-1)$. By considering this fact and equations (5.4) and (5.5), we find that

$$
\tilde{C}(X, Y) Z=\tilde{P}(X, Y) Z .
$$

Conversely, if the relation (5.6) holds, then from (5.4) and (5.5), we can easily obtain

$$
\tilde{S}(X, Z) Y-\tilde{S}(Y, Z) X=\frac{\tilde{r}}{n}\{g(Y, Z) X-g(X, Z) Y\},
$$

which is equivalent to

$$
\tilde{S}(X, Z) \eta(Y)-\tilde{S}(Y, Z) \eta(X)=\frac{\tilde{r}}{n}\{g(Y, Z) \eta(X)-g(X, Z) \eta(Y)\} .
$$

Putting $Y=\xi$ in last equation and using (2.1) and (3.12), we get

$$
\tilde{S}(X, Z)+2(n-1) \eta(Z) \eta(X)=\frac{\tilde{r}}{n}\{\eta(Z) \eta(X)-g(X, Z)\} .
$$

Let $\left\{e_{i}, i=1,2,3, \ldots, n\right\}$ be an orthonormal basis of the tangent space at any point of the manifold $M$. Setting $X=Z=e_{i}$ in the last equation and taking summation over $i, 1 \leq i \leq n$, we immediately get $\tilde{r}=-2 n(n-1)$ and hence from (5.4) and (5.5), we obtain (4.5). From the above discussion, we can state the following corollary:

Corollary 5.3. Let $M$ be an $n(>3)$-dimensional Kenmotsu manifold equipped with a semi-symmetric metric connection $\tilde{\nabla}$ has $\eta-$ parallel Ricci tensor for $\tilde{\nabla}$. Then the concircular and projective curvature tensors for $\tilde{\nabla}$ coincide if and only if the Ricci tensor $\tilde{S}$ is either Codazzi type or cyclic parallel.

From the Corollaries (4.7), (4.8) and (5.3), we observe the following:

Corollary 5.4. If the Ricci tensor $\tilde{S}$ of an $n(>3)$-dimensional Kenmotsu manifold $M$ endowed with a semi-symmetric metric connection $\tilde{\nabla}$ is $\eta$-parallel. Then the following results on $M$ are equivalent

(i) Ricci tensor $\tilde{S}$ is of Codazzi type,

(ii) Ricci tensor $\tilde{S}$ is cyclic parallel,

(iii) $\tilde{S}=-2(n-1) g(Y, Z)$,

(iv) $\tilde{C}(X, Y) Z=\tilde{P}(X, Y) Z$,

(v) $\tilde{r}=-2 n(n-1)$.

Let us suppose that the manifold $M$ equipped with a semi-symmetric metric connection $\tilde{\nabla}$ is either concircularly or projectively flat with respect to the connection $\tilde{\nabla}$, then in consequence of $(4.5),(5.4),(5.5)$ and (5.6), we find that

$$
\tilde{R}(X, Y) Z=-2\{g(Y, Z) X-g(X, Z) Y\},
$$

which shows that the manifold $M$ equipped with $\tilde{\nabla}$ is of constant curvature. Therefore we have:

Remark 5.5. The idea of constant curvature plays a central role in the theory of relativity and cosmology. The simplest cosmological model can be constructed by assuming that the universe is isotropic and homogeneous. This is known as cosmological principle. When we translated this principle to Riemannian geometry, professes that the three dimensional position space is a space of maximal symmetry [24], i.e., a space of constant curvature whose curvature depends upon time. The cosmological solutions of Einstein equations which contain a three dimensional space like surfaces of a constant curvature are the Robertson-Walker metric, while four dimensional space of constant curvature is the de Sitler model of the universe ([23], [24]).

In consequence of (3.8) and (5.7), we immediately get

$$
R(X, Y) Z=\{g(Y, Z) X-g(X, Z) Y\}+2\{\eta(X) \eta(Z) Y-\eta(Y) \eta(Z) X-\eta(X) g(Y, Z) \xi+\eta(Y) g(X, Z) \xi\},
$$

which shows that it is a certain class of generalized Sasakian space form (for instance, see [33], [34]). From (5.8), it is obvious that $f_{1}=1$, $f_{2}=0$ and $f_{3}=2$. Also, if we suppose that the manifold $M$ equipped with $\tilde{\nabla}$ satisfies (5.8), then equations (3.8), (5.4), (5.5) and (5.8) give $\tilde{C}=\tilde{P}=0$. Kim [34] proved that a generalized Sasakian-space form is conformally flat if and only if $f_{2}=0$. Thus from (5.8) and result of Kim, we have the following theorem:

Theorem 5.6. Let $M$ be an $n(>3)$-dimensional Kenmotsu manifold endowed with a semi-symmetric metric connection $\tilde{\nabla}$. Then the manifold is conformally flat if and only if it is either projectively or concircularly flat for $\tilde{\nabla}$.

Taking covariant derivative of (5.4) with respect to the semi-symmetric metric connection $\tilde{\nabla}$ along the vector field $W$, we get

$$
\left(\tilde{\nabla}_{W} \tilde{C}\right)(X, Y) Z=\left(\tilde{\nabla}_{W} \tilde{R}\right)(X, Y) Z-\frac{d \tilde{r}(W)}{n(n-1)}\{g(Y, Z) X-g(X, Z) Y\} .
$$

Let us suppose that the Kenmotsu manifold $M$ equipped with a semi-symmetric metric connection $\tilde{\nabla}$ has $\eta$-parallel Ricci tensor, i.e., $\left(\tilde{\nabla}_{X} \tilde{S}\right)(\phi Y, \phi Z)=0$. Thus from the Corollary 4.4, equation (5.9) assumes the form

$$
\left(\tilde{\nabla}_{W} \tilde{C}\right)(X, Y) Z=\left(\tilde{\nabla}_{W} \tilde{R}\right)(X, Y) Z .
$$

Before going to discuss our results, we define the following definitions as: 
Definition 5.7. An $n$-dimensional Kenmotsu manifold $M$ equipped with a semi-symmetric metric connection $\tilde{\nabla}$ is said to be a global symmetric Kenmotsu manifold with respect to the connection $\tilde{\nabla}$ if its non vanishing curvature tensor $\tilde{R}$ satisfies

$$
\left(\tilde{\nabla}_{W} \tilde{R}\right)(X, Y) Z=0,
$$

for arbitrary vector fields $X, Y, Z$ and $W$.

Definition 5.8. An $n$-dimensional Kenmotsu manifold $M$ equipped with a semi-symmetric metric connection $\tilde{\nabla}$ is said to be a global concircularly symmetric Kenmotsu manifold with respect to the connection $\tilde{\nabla}$ if its concircular curvature tensor $\tilde{C}$ satisfies

$$
\left(\tilde{\nabla}_{W} \tilde{C}\right)(X, Y) Z=0
$$

for arbitrary vector fields $X, Y, Z$ and $W$.

Definition 5.9. An $n$-dimensional Kenmotsu manifold $M$ equipped with a semi-symmetric metric connection $\tilde{\nabla}$ is said to be a globally $\phi$-symmetric Kenmotsu manifold with respect to the semi-symmetric metric connection $\tilde{\nabla}$ if its non vanishing curvature tensor $\tilde{R}$ satisfies

$$
\phi^{2}\left(\left(\tilde{\nabla}_{W} \tilde{R}\right)(X, Y) Z\right)=0,
$$

for arbitrary vector fields $X, Y, Z$ and $W$.

Definition 5.10. An $n$-dimensional Kenmotsu manifold $M$ equipped with a semi-symmetric metric connection $\tilde{\nabla}$ is said to be a globally $\phi$-concircularly symmetric Kenmotsu manifold with respect to the semi-symmetric metric connection $\tilde{\nabla}$ if its concircular curvature tensor $\tilde{C}$ satisfies

$$
\phi^{2}\left(\left(\tilde{\nabla}_{W} \tilde{C}\right)(X, Y) Z\right)=0,
$$

for all $X, Y, Z, W \in \chi(M)$.

In consequence of equation (5.10) and Definitions 5.7 and 5.8, we can observe the following:

Theorem 5.11. Let $M$ be an $n$-dimensional Kenmotsu manifold with a semi-symmetric metric connection $\tilde{\nabla}$ and the Ricci tensor $\tilde{S}$ of $M$ is $\eta$-parallel. Then $M$ is globally symmetric if and only if it is globally concircularly symmetric with respect to the connection $\tilde{\nabla}$.

Operating $\phi^{2}$ on both sides of (5.10), we have

$$
\phi^{2}\left(\tilde{\nabla}_{W} \tilde{C}\right)(X, Y) Z=\phi^{2}\left(\tilde{\nabla}_{W} \tilde{R}\right)(X, Y) Z .
$$

Thus, with the help of above equation and Definitions 5.3 and 5.4, we can state:

Theorem 5.12. If an $n$-dimensional Kenmotsu manifold $M$ equipped with a semi-symmetric metric connection $\tilde{\nabla}$ has $\eta-$ parallel Ricci tensor $\tilde{S}$, then the manifold $M$ to be globally $\phi$-symmetric if and only if it is globally $\phi$-concircularly symmetric.

It is observed that a globally $\phi$-concircularly symmetric Kenmotsu manifold $M$ equipped with a semi-symmetric metric connection $\tilde{\nabla}$ is an $\eta$-Einstein manifold. Thus, by considering this fact and Theorem 5.12, we have

Corollary 5.13. If an $n$-dimensional Kenmotsu manifold $M$ endowed with a semi-symmetric metric connection $\tilde{\nabla}$ has $\eta$-parallel Ricci tensor $\tilde{S}$, then it is an $\eta-$ Einstein manifold.

\section{Example}

In this section, we construct an example of the Kenmotsu manifold admitting a semi-symmetric metric connection and after that we validate our results.

\section{Example 6.1. Let}

$$
M^{3}=\left\{(x, y, z) \in \mathbb{R}^{3}: x, y, z(\neq 0) \in \mathbb{R}\right\},
$$

be a three dimensional smooth manifold, where $(x, y, z)$ denotes the standard coordinate of a point in $\mathbb{R}^{3}$. Let us suppose that

$$
e_{1}=e^{z}\left(\frac{\partial}{\partial x}+\frac{\partial}{\partial y}\right), e_{2}=e^{z} \frac{\partial}{\partial y}, e_{3}=-\frac{\partial}{\partial z}
$$

be a set of linearly independent vector field at each point of the manifold $M^{3}$ and therefore it form a basis for the tangent space $\chi\left(M^{3}\right)$. We also define the Riemannian metric $g$ of the manifold $M^{3}$ as $g\left(e_{i}, e_{j}\right)=\delta_{i j}$, where $\delta_{i j}$ denotes the Kronecker delta and $i, j=1,2,3$. Let us consider the $1-$ form $\eta$ defined by $\eta(Z)=g\left(Z, e_{3}\right)$ for any $Z \in \chi\left(M^{3}\right)$ and a tensor field $\phi$ of type $(1,1)$ defined by

$$
\phi\left(e_{1}\right)=-e_{2}, \phi\left(e_{2}\right)=e_{1}, \phi\left(e_{3}\right)=0 .
$$

By the linearity properties of $\phi$ and $g$, we can easily verify the following relations

$$
\phi^{2} X=-X+\eta(X) e_{3}, \quad \eta\left(e_{3}\right)=1, \quad g(\phi X, \phi Y)=g(X, Y)-\eta(X) \eta(Y)
$$

for arbitrary vector fields $X, Y \in \chi\left(M^{3}\right)$. This shows that $\xi=e_{3}$ and the structure $(\phi, \xi, \eta, g)$ defines an almost contact metric structure on $M^{3}$. If $\nabla$ represents the Levi-Civita connection with respect to the Riemannian metric g, then with help of above, we can easily calculate that

$$
\left[e_{1}, e_{2}\right]=0, \quad\left[e_{1}, e_{3}\right]=e_{1}, \quad\left[e_{2}, e_{3}\right]=e_{2} .
$$


We recall the Koszul's formula as

$$
2 g\left(\nabla_{X} Y, Z\right)=X g(Y, Z)+Y g(X, Z)-Z g(X, Y)-g(X,[Y, Z])-g(Y,[X, Z])+g(Z,[X, Y])
$$

for arbitrary vector fields $X, Y, Z \in \chi\left(M^{3}\right)$. It is obvious from Koszul's formula that

$$
\nabla_{e_{1}} e_{1}=-e_{3}, \quad \nabla_{e_{1}} e_{2}=0, \quad \nabla_{e_{1}} e_{3}=e_{1}, \quad \nabla_{e_{2}} e_{1}=0, \quad \nabla_{e_{2}} e_{2}=-e_{3}, \quad \nabla_{e_{2}} e_{3}=e_{2}, \quad \nabla_{e_{3}} e_{1}=0, \quad \nabla_{e_{3}} e_{2}=0, \quad \nabla_{e_{3}} e_{3}=0 .
$$

From the above calculations, we can observe that $\nabla_{X} \xi=X-\eta(X) \xi$, for $\xi=e_{3}$. Thus the manifold $\left(M^{3}, g\right)$ is a Kenmotsu manifold of dimension 3 and the structure $(\phi, \eta, \xi, g)$ denotes the Kenmotsu structure on the manifold $M^{3}$.

It is obvious from the above results that

$$
\begin{array}{lrrr}
R\left(e_{1}, e_{2}\right) e_{3}=0, & R\left(e_{1}, e_{3}\right) e_{3}=-e_{1}, \quad R\left(e_{3}, e_{2}\right) e_{2}=-e_{3}, & R\left(e_{3}, e_{1}\right) e_{1}=-e_{3}, \quad R\left(e_{2}, e_{1}\right) e_{1}=-e_{2}, \\
R\left(e_{2}, e_{3}\right) e_{3}=-e_{2}, \quad R\left(e_{1}, e_{2}\right) e_{2}=-e_{1}, \quad R\left(e_{3}, e_{1}\right) e_{2}=0, & S\left(e_{1}, e_{1}\right)=-2, & S\left(e_{2}, e_{2}\right)=-2, \\
S\left(e_{3}, e_{3}\right)=-2, \quad S\left(\phi e_{1}, \phi e_{1}\right)=-2, \quad S\left(\phi e_{2}, \phi e_{2}\right)=-2, & S\left(\phi e_{3}, \phi e_{3}\right)=0, & S\left(\phi e_{i}, \phi e_{j}\right)=0, \text { for all } i, j=1,2,3(i \neq j) .
\end{array}
$$

From the above relations, we can easily calculate that $\left(\nabla_{X} S\right)\left(\phi e_{i}, \phi e_{j}\right)=0$ for all $X \in \chi\left(M^{3}\right)$ and $i, j=1,2,3$. Hence the manifold $M^{3}$ is $\eta$-parallel.

In consequence of (3.3) and above results, we can find that

$$
\tilde{\nabla}_{e_{1}} e_{1}=-2 e_{3}, \quad \tilde{\nabla}_{e_{1}} e_{2}=0, \quad \tilde{\nabla}_{e_{1}} e_{3}=2 e_{1}, \quad \tilde{\nabla}_{e_{2}} e_{1}=0, \quad \tilde{\nabla}_{e_{2}} e_{2}=-2 e_{3}, \tilde{\nabla}_{e_{2}} e_{3}=2 e_{2}, \quad \tilde{\nabla}_{e_{3}} e_{1}=0, \quad \tilde{\nabla}_{e_{3}} e_{2}=0, \quad \tilde{\nabla}_{e_{3}} e_{3}=0
$$

and also the components of torsion tensor $\tilde{T}$ are

$$
\tilde{T}\left(e_{i}, e_{i}\right)=\tilde{\nabla}_{e_{i}} e_{i}-\tilde{\nabla}_{e_{i}} e_{i}-\left[e_{i}, e_{i}\right]=0, \text { for } i=1,2,3 \quad \text { and } \quad \tilde{T}\left(e_{1}, e_{2}\right)=0, \quad \tilde{T}\left(e_{1}, e_{3}\right)=e_{1}, \quad \tilde{T}\left(e_{2}, e_{3}\right)=e_{2} .
$$

These equations show that $\tilde{T} \neq 0$ and therefore by equation (3.1), we can say that the linear connection defined in (3.3) is a semi-symmetric connection on $\left(M^{3}, g\right)$. By the straight forward calculation, we can also find

$$
\left(\tilde{\nabla}_{e_{1}} g\right)\left(e_{i}, e_{j}\right)=0, \quad\left(\tilde{\nabla}_{e_{2}} g\right)\left(e_{i}, e_{j}\right)=0, \quad\left(\tilde{\nabla}_{e_{3}} g\right)\left(e_{i}, e_{j}\right)=0
$$

for all $i, j=1,2,3$. This demonstrates that the equation (3.2) holds on $M^{3}$ and hence the linear connection defined in (3.3) is a semisymmetric metric connection on $M^{3}$. Thus we can say that the manifold $\left(M^{3}, g\right)$ be a three dimensional Kenmotsu manifold equipped with a semi-symmetric metric connection $\tilde{\nabla}$ defined in (3.3).

With the help of above discussions, we can calculate the curvature and Ricci tensors with respect to the semi-symmetric metric connection $\tilde{\nabla}$ as

$$
\begin{aligned}
& \tilde{R}\left(e_{1}, e_{2}\right) e_{3}=0, \tilde{R}\left(e_{1}, e_{3}\right) e_{3}=-2 e_{1}, \tilde{R}\left(e_{3}, e_{2}\right) e_{2}=-2 e_{3}, \tilde{R}\left(e_{3}, e_{1}\right) e_{1}=-2 e_{3}, \tilde{R}\left(e_{2}, e_{1}\right) e_{1}=-4 e_{2}, \tilde{R}\left(e_{2}, e_{3}\right) e_{3}=-2 e_{2}, \\
& \tilde{R}\left(e_{1}, e_{2}\right) e_{2}=-4 e_{1}, \tilde{R}\left(e_{3}, e_{1}\right) e_{2}=0, \tilde{S}\left(e_{1}, e_{1}\right)=-6, \tilde{S}\left(e_{2}, e_{2}\right)=-6, \tilde{S}\left(e_{3}, e_{3}\right)=-4, \tilde{r}=-16
\end{aligned}
$$

and other components can be calculated by symmetric and skew-symmetric properties. We can easily observe that the equations [(3.8) (3.14)] are verified. Also,

$$
\tilde{S}\left(\phi e_{1}, \phi e_{1}\right)=-6, \tilde{S}\left(\phi e_{2}, \phi e_{2}\right)=-6, \tilde{S}\left(\phi e_{3}, \phi e_{3}\right)=0, \quad \tilde{S}\left(\phi e_{i}, \phi e_{j}\right)=0, \text { for all } i, j=1,2,3(i \neq j) .
$$

It is clear from the above discussions that $\left(\tilde{\nabla}_{X} \tilde{S}\right)\left(\phi e_{i}, \phi e_{j}\right)=0$ for all $X \in \chi\left(M^{3}\right)$ and $i, j=1,2,3$. Hence the manifold $M^{3}$ equipped with a semi-symmetric metric connection $\tilde{\nabla}$ has $\eta$-parallel Ricci tensor for the connection $\tilde{\nabla}$. From the above discussions, we come to the conclusion:

"If the manifold $M^{3}$ has $\eta$-parallel Ricci tensor with respect to Levi-Civita connection $\nabla$, then it contains also $\eta$-parallel Ricci tensor with respect to the semi-symmetric metric connection $\tilde{\nabla} "$.

Hence the statement of the Theorem 4.2.

It is obvious from the above relations that $\tilde{r}=-16$ (constant) and hence the Corollary 4.4 is satisfied on $M^{3}$.

\section{References}

[1] Robert Osserman, Curvature in the Eighties, The American mathematical monthly 97 (8), (1990), 731-756.

[2] É. Cartan, Surune classe remarquable d'espaces de Riemannian, Bull. Soc. Math. France 54 (1926), $214-264$.

[3] É. Cartan, Le cons sur la géeométrie des espaces de Riemann, 2nd ed., Paris, 1946.

[4] B. O' Neill, Semi-Riemannian geometry with applications to the relativity, Academic Press, New York- London, 1983.

[5] M. M. Boothby and R. C. Wong, On contact manifolds, Anna. Math. 68 (1958), 421-450.

[6] S. Sasaki and Y. Hatakeyama, On differentiable manifolds with certain structures which are closely related to almost contact structure, Tohoku Math. J. 13 (1961), 281-294.

[7] K. Kenmotsu, A class of almost contact Riemannian manifolds, Tohoku Math. J. 24 (1972), 93-103.

[8] S. K. Chaubey and A. A. Shaikh, On 3-dimensional Lorentzian concircular structure manifolds, Commun. Korean Math. Soc., 33 (2018) https://doi.org/10.4134/CKMS.c180044.

[9] S. K. Chaubey and R. H. Ojha, On the m-projective curvature tensor of a Kenmotsu manifold, Differential Geometry - Dynamical Systems 12 (2010), 52-60.

[10] S. K. Chaubey, S. Prakash and R. Nivas, Some properties of m-projective curvature tensor in Kenmotsu manifolds, Bulletin of Math Analysis and Applications 4 (2012), 48-56.

[11] S. K. Chaubey and C. S. Prasad, On generalized $\phi$-recurrent Kenmotsu manifolds, TWMS J. App. Eng. Math. 5 (1) (2015), 1-9.

[12] S. K. Yadav, S. K. Chaubey and D. L. Suthar, Certain results on almost Kenmotsu $(\kappa, \mu, v)-$ spaces, Konuralp Journal of Mathematics 6 (1) (2018) 128-133

[13] S. K. Chaubey, A. C. Pandey, N. V. C. Shukla, Some properties of Kenmotsu manifolds admitting a semi-symmetric non-metric connection, arXiv:1801.03000v1 [math.DG] 9 Jan 2018.

[14] S. K. Chaubey and R. H. Ojha, On a semi-symmetric non-metric connection, Filomat 26 (2) (2012), 269-275.

[15] A. Taleshian and A. A. Hosseinzadeh, Some curvature properties of Kenmotsu manifolds, Proc. of the Nat. Academy of Sci. 85 (2015), $407-413$. 
[16] A. Basari and C. Murathan, On generalized $\phi$-recurrent Kenmotsu manifolds, Fen Derg. 3 (1) (2008), 91-97.

[17] H. Ozturk, N. Aktan and C. Murathan, On $\alpha$-Kenmotsu manifolds satisfying certain conditions, Applied sciences 12 (2010), $115-126$.

[18] K. Yano, Concircular geometry I, Concircular transformation, Proc. Imp. Acad. Tokyo 16 (1940), 195-200.

[19] K. Yano, Concircular geometry I, Imp. Acad. Sci. of Japan 16 (1940), 195-200.

[20] A. Friedmann and J. A. Schouten, Über die geometrie der halbsymmetrischen übertragung, Math. Zeitschr 21 (1924), $211-223$.

[21] H. A. Hayden, Subspaces of a space with torsion, Proc. London Math. Soc. 34 (1932), 27-50.

[22] K. Yano, On semi-symmetric metric connection, Rev. Roumaine de Math. Pures et Appl. 15 (1970), 1579-1586.

[23] J. V. Narlikar, General relativity and gravitation, The Macmillan co. of India, 1978.

[24] H. Stephani, General relativity-An introduction to the theory of gravitational field, Cambridge Univ. Press, Cambridge, 1982.

[25] D. E. Blair, Contact manifolds in Riemannian Geometry, Lecture Notes in Mathematics, 509, Springer-Verlag, Berlin, 1976.

[26] M. Kon, Invariant submanifolds in Sasakian manifolds, Math. Ann. 219 (1976), 277-290.

[27] S. K. Chaubey and A. Kumar, Semi-symmetric metric T-connection in an almost contact metric manifold, International Mathematical Forum 5 (23) (2010), 1121-1129.

[28] A. Sharfuddin and S. I. Husain, Semi-symmetric metric connections in almost contact manifolds, Tensor N. S. 30 (1976), $133-139$.

[29] T. Imai, Notes on semi-symmetric metric connections, Tensor N. S. 24 (1972), 293-296.

[30] R. S. Mishra and S. N. Pandey, Semi-symmetric metric connections in an almost contact manifold, Indian J. Pure Appl. Math. 9 (6) (1978), 570-580.

[31] A. Berman, Concircular curvature tensor of a semi-symmetric metric connection in a Kenmotsu manifold, Thai J. of Mathematics 13 (1) (2015), 245-257.

[32] Sunil Yadav and D. L. Suthar, On three-dimensional quasi-Sasakian manifolds admitting semi-symmetric metric connection, International Journal of Physical Sciences 8 (17) (2013), 754-758.

[33] P. Alegre, D. E. Blair and A. Carriazo, Generalized Sasakian Space forms, Israel J. of Math. 141 (2004), 157-183.

[34] U. K. Kim, Conformally flat generalized Sasakian-space forms and locally symmetric generalized Sasakian-space forms, Note Mat. 26 (2006), $55-67$.

[35] T. Takahashi, Sasakian $\phi$-symmetric spaces, Tohoku Math. J. 29 (1977), 91-113. 